\title{
Cementless unicompartmental knee replacement allows early return to normal activity
}

Benjamin Panzram, Ines Bertlich, Tobias Reiner, Tilman Walker, Sébastien Hagmann and Tobias Gotterbarm ${ }^{*}$ (B)

\begin{abstract}
Background: Physical activity and regular participation in recreational sports gain importance in patients' lifestyle after knee arthroplasty. Cementless unicompartimental Knee replacement with the Oxford System has been introduced into clinical routine. Currently there is no data reporting on the physical activity, return to sports rate and quality of live after medial cementless Oxford Unicompartimental Knee Replacement (OUKR).

Methods: This retrospective cohort study reports on the functional outcome of the first 27 consecutive patients (30 knees) that were consecutively treated with a cementless medial OUKR between 2007 and 2009 in our hospital. Physical activity and quality of life were measured using the Tegner-Score, the UCLA-Activity Score, the Schulthess Clinical Activity Questionnaire and the SF-36 Score. The patients' satisfaction with the outcome was measured using a visual analogue scale.
\end{abstract}

Results: Mean age at surgery was 62.5 years. Patients showed a rapid recovery with 17 out of 27 patients returning to sports within 3 months, 24 within 6 months after surgery. The Return-to-activity-rate was 100\%. 10 out of 27 patients showed a high activity level (UCLA $\geq 7$ points) with a mean postoperative UCLA-Score of 6.1 points.

Conclusions: Patients recover rapidly after cementless OUKR with a return to sports rate of $100 \%$ and patients are able to participate in high impact sports disciplines.

Keywords: Cementless UKR, OUKR, Oxford medial, Physical activity, Sports, Return-to-activity, Knee arthroplasty

\section{Background}

As life expectancy is increasing and the incidence of osteoarthritis (OA) rises with age, there is a high demand of joint replacement for people of middle and advanced age. While the mean age of patients receiving UKR is 63.6 years, several studies show that patients perform high levels of activities up until after 70 years of age [1-3].

Therefore, physical functioning and participation in sports after surgery are important outcome measures of a successful joint replacement. To allow high levels of activity, UKR provides more physiological knee kinematics, a higher range of motion, a more natural perception of the knee, shorter hospital stay, faster recovery with a lower rate of complication when compared to Total

\footnotetext{
* Correspondence: Tobias.Gotterbarm@med.uni-heidelberg.de Clinic of Orthopaedic and Trauma Surgery, University of Heidelberg, Schlierbacher Landstr. 200a, 69118 Heidelberg, Germany
}

Knee Replacement [4-6]. UKR has shown to have excellent long-term survival rates compared to TKR [7-9]. As the cemented UKR is a reliable treatment option for antero medial OA, its' widespread use has been recommended for the elderly as well as for younger OA patients (e.g. patients $<60$ years) $[4,10-13]$.

For further improvement of the clinical outcome, implant survival and to eliminate complications associated with cementation, a cementless medial OUKR has been developed. Cementless fixation may offer several advantages compared to cemented OUKR. Reduced operation time, the absence of possible cement related tissue reactions, inefficient cementation which might influence the fixation or lead to early wear, clinical symptoms or even revision due to foreign bodies. Furthermore, radiographic radiolucent lines are less common suggesting a superior biological fixation [14]. Especially for patients at younger age with higher demands of sports activity 
these benefits seem to be desirable. However, cementless fixation is associated with a higher risk of intra and postoperative tibial plateau fractures and tibial valgus subsidence. Particularly an extended sagittal saw cut, a low bone density might lower the fracture load which may lead to tibial plateau fractures in combination with the firm impaction to achieve the desired press fit [15]. Valgus subsidence in cementless OUKR may be caused by extensive vertical saw cuts and or laterally implanted femoral components, causing impingement of the inlay against the medial tibial wall during flexion [16].

So far, there are promising short- to medium-term results published by the developing centres as well as registry data, indicating good clinical outcome and survival rates with a lower revision rate compared to the cemented version [17-20]. So far there are no published data reporting on the physical activity level after cementless OUKR. We therefore report in this study on the physical activity, return to sports rate and quality of life of our first consecutive 30 cementless medial OUKR.

\section{Methods}

In this retrospective study we evaluated the first 27 patients (30 knees) who were treated consecutively with a cementless medial OUKR in our hospital. The study was assessed and approved by the ethics committee of the University of Heidelberg (S-546/2013). Surgery was performed by three experienced surgeons between 2007 and 2009 using the Oxford III System. The patient cohort was described in our previous work analysing the incidence of radiolucent lines in cementless fixation [21].

Patients were examined before surgery and at final follow-up. The level of physical activity before and after surgery was measured using Tegner and UCLA Activity Score. Detailed information about physical activity was obtained using the Schulthess Clinical Activity Questionnaire, which compares the state at follow-up with the last time point before the onset of OA symptoms. The SF-36 Score determines the self-perception of the patients' quality of life, compared to a healthy cohort and a standard group suffering from osteoarthritis/ rheumatoid arthritis. The patient's satisfaction with the operated knee was measured using a visual analogue scale (0-10).

The indication for operation in all cases was anteromedial osteoarthritis (OA) with intact lateral knee compartment. The anterior cruciate ligament (ACL) and the collateral ligaments were intact and the varus deformity was fully correctable manually. A flexion deformity $>15^{\circ}$ or previous osteotomy were contraindications for the procedure, while cartilage loss in the femoro-patellar joint, age and obesity were not considered as contraindications [22]. Indications were concordant with the recommendations by Goodfellow et al. [23]. After surgery, the patients followed a three-week rehabilitation scheme with full weight-bearing.

\section{Data analysis}

SPSS Version 21 was applied to analyse the data. We used the Pearson's Chi Square Test for categorial and ordinal variables. Comparison of Pre-and post-operative scores were performed utilising the Wilcoxon signedrank test. To compare differences between two independent groups with ordinal or continuous variables we used the Mann-Whitney $U$ test. $P$-values of 0.05 or smaller were considered as significant.

\section{Results}

\section{Demographics and study group}

The study group consisted of the first 27 consecutive patients (15 male, 12 female, 30 knees) that were consecutively treated with cementless OUKR in our institution. Patient age at surgery ranged from 49 to 76 years with a mean of 62.5 years. Mean follow-time after surgery was 60 months, raging from 47 to 69 months (SD 8.3).

No patient died during follow up. Overall 3 knees were excluded from the study. In one case the reason was a major deviation from the recommended surgical technique. One patient suffered a periprosthetic tibial fracture within the first month after initial operation with consecutive revision of the tibial component to a cemented version and ORIF. The third patient was excluded after total knee replacement following progressive OA of the lateral and the patellofemoral joint (PFJ). The remaining 27 knees (24 patients) were included in the clinical and functional assessment. We observed one reoperation due to dislocation of the mobile-bearing 21 months postoperative and consecutive exchange of the inlay to a thicker one. In one case OA of the PFJ resulted in additional patello femoral arthroplasty (PFA).

\section{Return to activity}

Twenty-four out of 27 patients were physically active before surgery and all have returned to sports at finalfollow up (see Table 1).

Seventeen patients (18 knees) returned to sports within 3 months after surgery (see Fig. 1). There were no age- and gender-related differences.

Most popular activities before and after surgery were cycling, hiking and long walks (see Fig. 2). Altogether, 18 types of sport were performed pre- or postoperatively. There were 5 types of high-impact sports practised before the onset of OA symptoms as well as after surgery. There was a notable shift from giving up sports such as soccer or jogging and starting volleyball and mountaineering. The main cause for the change was "pain" (3 patients, 4 knees). The Change did not reach statistical significance. $(p=0.202)$. 
Table 1 Return to activity

\begin{tabular}{|c|c|c|c|c|}
\hline & & After surgery & & Total \\
\hline & & $\begin{array}{l}\text { Active (patients/ } \\
\text { knees) }\end{array}$ & $\begin{array}{l}\text { Inactive (patients/ } \\
\text { knees) }\end{array}$ & $\begin{array}{l}\text { (patients/ } \\
\text { knees) }\end{array}$ \\
\hline Before surgery & Active & $24 / 27$ & $0 / 0$ & $24 / 27$ \\
\hline & Inactive & $2 / 2$ & $1 / 1$ & $3 / 3$ \\
\hline Total & & $26 / 29$ & $1 / 1$ & $27 / 30$ \\
\hline
\end{tabular}

\section{Amount of activities}

There was no significant difference $(p=0.132)$ regarding the number of sports disciplines before the onset of OA symptoms and after surgery. During sports, 20 patients (22 knees) did not experience pain and 4 patients (4 knees) practised although feeling pain. One patient (one knee) did not participate in sports after surgery.

The quantitative assessment of sports participation was done using either the total number of patients that practised sports at least three times per week or the number of patients that practised at least $1 \mathrm{~h}$ per training session. There was no statistically significant difference comparing the state before OA onset and at follow-up in both parameters $(p=0.146)$.

While practicing sports, 19 patients (19 knees) felt excellent and did not report on any limitation or discomfort. Three patients (four knees) described a feeling of insecurity or fear of damaging the knee implant, three patients (three knees) felt they had a limit in the range of motion, and two patients each (two knees each) reported that they were not in a good physical condition or had a limited general flexibility.

\section{Scores and satisfaction}

All 24 patients (27 knees) showed a significant improvement in both Tegner and UCLA-Scores after surgery ( $p=0.042$ each, see Fig. 3). UCLA-Score was 4.9 (SD 2.3) preoperatively and increased significantly to 6.1 after surgery (SD 1.8), with a mean change of 1.2 points (SD 1.8). Tegner Score improved by 0.5 points (SD 0.2) from 2.9 points before surgery (SD

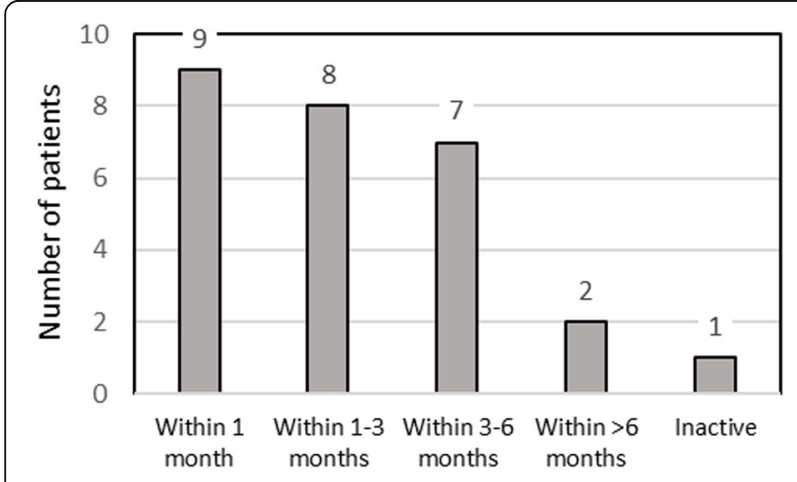

Fig. 1 Return to activity
$1.4)$ to 3.4 points postoperatively (SD1.0). We classified the pre- and postoperative UCLA-Scores into three categories: $\geq 7$ points: high activity levels, 4-6 points: moderate activity and $\leq 3$ points: low activity [24]. At follow-up, 10 patients (11 knees) were highly active and 14 patients (12 knees) showed moderate activity. Three patients ( 4 knees) showed low activity levels.

The SF-36 Score showed high score values in all patients with cementless OUKR at final follow-up (see Fig. 4). The Results compared to the two reference groups are shown in Fig. 4. Overall 23 patients were extremely and very satisfied with the outcome, 4 patients were satisfied.

\section{Discussion}

This retrospective study assessed the physical activity and satisfaction in the first 27 consecutive patients (15 male, 12 female, 30 knees) that were treated with cementless OUKR in our institution between 2007 and 2009. Mean follow-up time was 60.0 months (47-69; SD 8.3) and mean age at surgery was 62.5 years (range $49-76$ ).

Our main finding was that patients showed a high level of activity after cementless OUKR. Return-toactivity rate was $100 \%$ and the extent of activity did not differ from the time point before the onset of OA symptoms. The postoperative mean UCLA of 6.1 points and the predominant number of patients achieving 7 points or more in the UCLA-Score (10/26 patients) displayed that patients after cementless medial OUKR were able to reach a high level of impact sports.

In a meta-analysis by Witjes et al., the postoperative activity in 8 studies of patients receiving cemented UKR and 13 studies of patients receiving TKR was analysed. They found that the postoperative return-to-activity-rate as well as the level of activity were higher after cemented UKR than after TKR. They reported return-to-activityrates between 75 - >100\% in the UKR group and 36$89 \%$ in the TKR group. Our results indicate that cementless OUKR also allows patients high return- to-activityrates compared to cemented UKR [25]. Although other authors presume that the effects of the learning curve, regarding the implantation of Oxford UKR, might affect the postoperative outcome and therefore the physical activity, our results show similar physical activity compared to cemented implantation [26, 27].

Our return-to-activity-rate showed higher values compared to studies about Oxford medial UKR, which range between $80.1 \%$ and $97 \%$ [3, 11, 28, 29]. In a retrospective study on the activity after cemented OUKR with a follow-up of 4.2 years, Pietschmann et al. reported a return-to-activity rate of $80.1 \%$ [3]. Possible reasons include the larger patient cohort and the high age of 


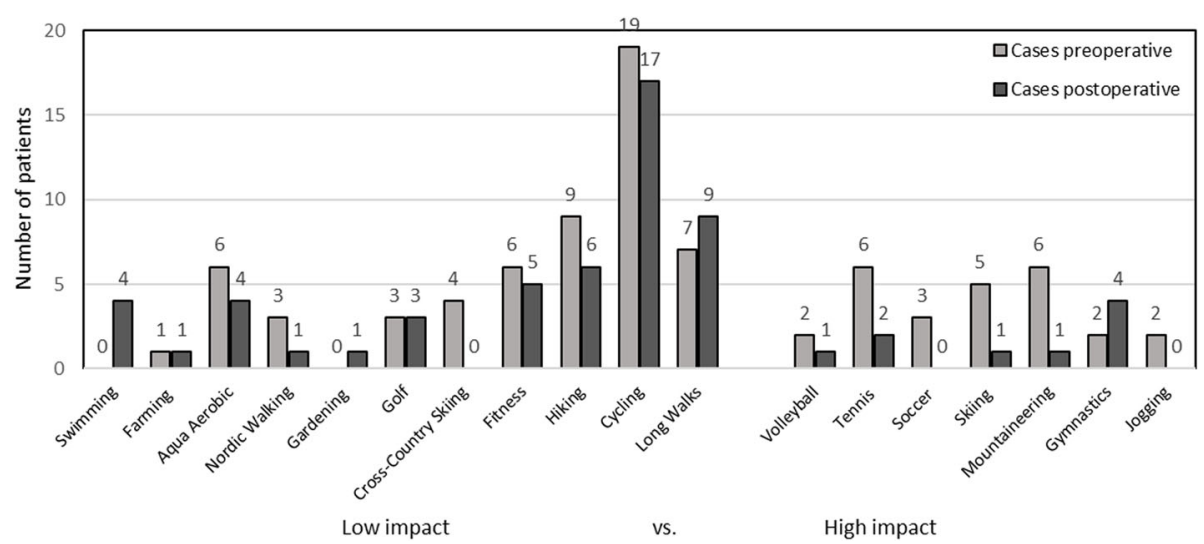

Fig. 2 Sports and activities

treated patients (131 patients with a mean age of 65.3 years compared to 27 patients with a mean age of 62.5 years). They split the patient collective into an active and an inactive group and found the active group to be significantly younger than the inactive group. They referred to the results of the "German Health Survey" 2006, which showed that activity decreased significantly in patients over 70 years of age. At five-year follow-up, the average patient in our study was 67.5 years old, compared to 69.5 years in Pietschmann's study. Walker et al. reported a return-to-activity rate of $93 \%$ in patients of sixty years or younger after medial UKR. Almost two thirds reached postoperative UCLA-Scores $>7$ [11]. This matches the excellent outcome of our study with almost half of the collective being younger than sixty years. However, we did not detect a significant difference in the activity levels of patients older and younger than median age (data not shown).

In our study, patients did recover quickly after cementless OUKR, which supports literature findings

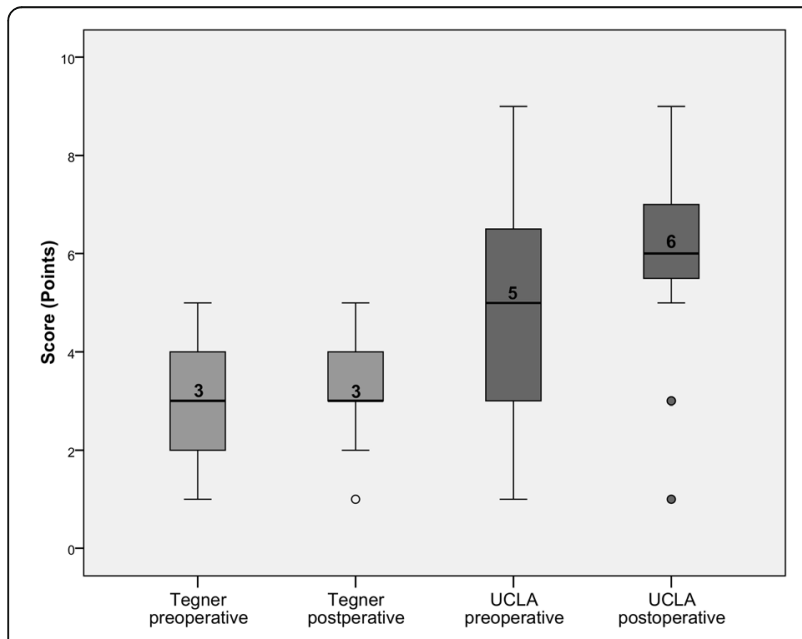

Fig. 3 Tegner- and UCLA-Score before and after surgery
$[25,30]$. Price et al. showed minimal-invasive OUKR patients to recover twice as fast as standard incision UKR patients and three times as fast as TKR patients [31]. More than $60 \%$ of the patients in our study had picked up sports already during 3 months after surgery and $90 \%$ within the first 6 months.

Another finding of the present study is the high rate of patients without pain during sports (22 out of 26, 85\%). Others have reported on the amount of patients being pain-free during activity ranging between $57 \%$ and $76 \%$ after cemented OUKR $[11,29]$. A possible explanation for these different findings might be a shorter follow-up time of approximately 2 years compared to the five-year follow-up in our study.

In our study, UCLA-Score improved significantly from 4.9 points preoperatively to 6.1 points postoperatively $(p=0.042)$. This matches the findings of other studies: Fisher et al. reported an improvement from 4.2 to 6.5 points [28]. Generally, postoperative UCLAScores range from 6.1 to 6.8 points $[11,25,32,33]$. Tegner-Score values are the only activity-related item that was published so far on cementless medial OUKR. In the randomized controlled trial, Pandit et al. compared cementless- with cemented OUKR. They reported an improvement from 1.9 points preoperatively to 3.1 points 2 years after implantation and 2.9 points at five-year follow-up in the cementless group. At 2 years follow-up, the Tegner-Score was significantly higher in the cementless group compared to the cemented group, but the difference did not persist until 5 years after surgery [19]. Tegner Score in our study was 2.9 points preoperatively and 3.4 points postoperatively at a mean follow up of 5 years, with a significant improvement $(p=0.042)$.

Concerning the extent, frequency and length of activities in our study, we found that there was no significant decrease after surgery compared to the time before the onset of OA symptoms. Our findings match the results 

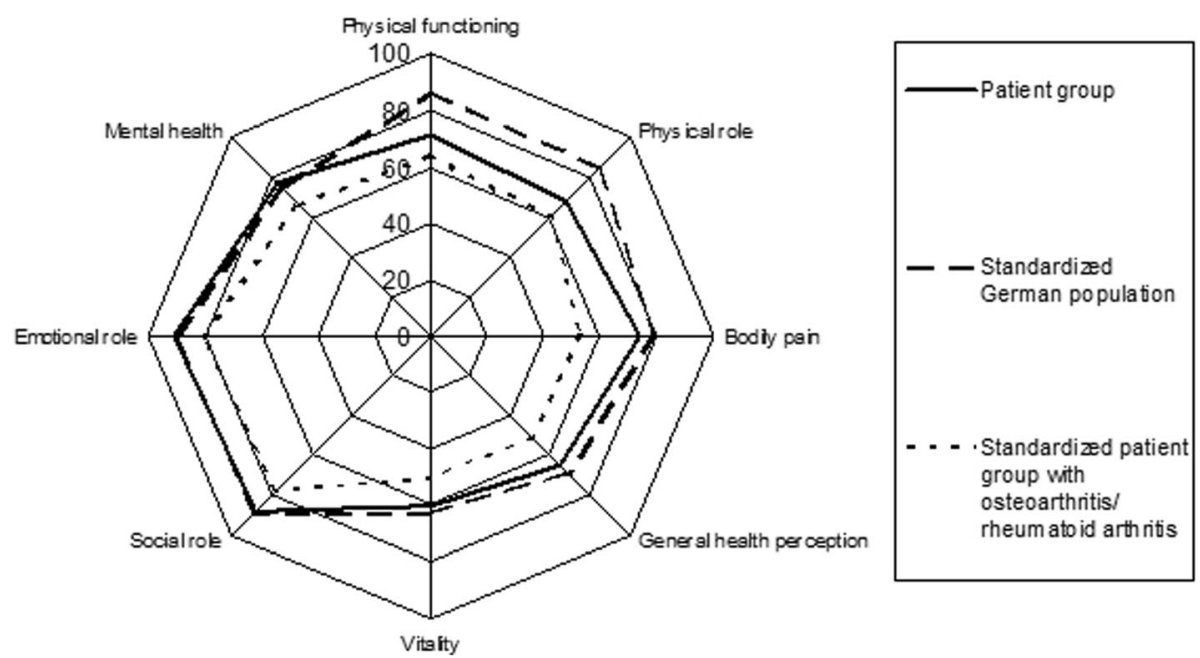

Fig. 4 SF-36 Score

of other authors assessing activity after cemented OUKR as well as activity after cementless Total Hip Arthroplasty [11, 34].

Sports were divided into high-impact (with high peak loads in the joints) and low-impact forms (with constant low joint loads). In contrast to other authors assessing postoperative activity after UKR, we did not find a significant increase or decrease in any type of sports after surgery. There are several authors reporting a significant decrease of high-impact sports after surgery, while lowimpact sports tend to increase [2, 3, 11, 35]. The reason for this is might be attributed to multiple causes such as the surgeon's recommendations, lack of function, feeling of insecurity, pain, comorbidities etc. Asked for their reasons to abandon high-impact sports, the majority of patients in a study by Walker et al. named "precaution" (59\%) and "less motivation" (20\%), while pain only followed fourth with 9\% [30]. Although there is no final conclusion on the best type of sports for patients after joint replacement, there seems to be a general consensus from surgeons to discourage patients from high-impact sports such as soccer and tennis [36]. Supporting this position, there is indication that high-impact sports lead to high joint loads and can thereby increase implant wear followed by a higher rate of complications and revisions [36, 37]. Mobile-bearing devices such as the cementless OUKR are known to minimize wear due to the fully congruent mobile bearing [38, 39]. In accordance with these findings, although the postoperative level of activity was high, there were no revisions due to implant wear in this five-year follow-up study. Pietschmann et al. report that although they noticed a significant decrease in high-impact sports, they did not detect a correlation between high impact sports and complication. General activity is necessary to maintain cardiovascular fitness and bone density. There are several studies indicating that bone density depends on frequency of activity as well as on the imposed skeletal forces, indicating that impact up to a certain level has positive effects on bone density and should not generally be discouraged [40-42].

Assessing the quality of life, our patients reported excellent results. Regarding the physical dimensions, they accomplished higher values than the reference group suffering from OA/ rheumatoid arthritis. In the emotional-social domains of SF-36, they reached the same or better scores than the healthy reference population. Naal et al. compared the findings of their population to a matched reference group and they achieved significantly higher scores in every domain [2]. A possible explanation for the higher scores could be higher preoperative scores of their collective or the short time between surgery and questioning (18 months). Recently operated patients tend to remember their state before surgery more easily. Both studies showed high SF-36 Scores and were comparable with the findings of Walker et al., who investigated activity after cemented OUKR in patients $\leq 60$ years of age [11].

In the present study, in accordance with literature, patients reported a high satisfaction rate with the outcome of the joint replacement $[3,11]$.

Superior radiological osseointegration and no cement associated complications seem to be a clear advantage of cementless fixation, especially desirable for young and active patients. However, possible early intra and postoperative complications associated with cementless OUKR like periprosthetic fractures and valgus subsidence might impair early return to sports and activity. Overall these complications are rare and appear to be rather influenced by mistakes in the surgical technique $[15,16]$. 
Main limitation of this study is the small number of patients. We did not collect preoperative SF-36 data which makes the efficacy of cementless OUKR hard to compare to other author's findings, but as we aimed to compare the postoperative quality of life with a healthy reference group, we think that the conclusions are not impaired.

Furthermore, although the scores and questionnaires used in our study (Tegner, UCLA, Schulthess Clinical Activity Questionnaire) are validated for the evaluation of physical activity, it is a difficult to quantify and compare the results as many parameters can only be answered using a free text, which makes subcategorization difficult. Activity cannot be reduced to one parameter only, thus comparison of many aspects of activity is necessary. However, patients' subjective perceptions of sports capability may be an outcome measure that outweighs the supposed objective parameters of physical activity.

Another major weakness of this study besides the retrospective design might be selection bias since the patients were recruited within a 3-year time period (2007-2009) between the first and last inclusion.

The strength of this study is its detailed information at a mean follow-up of 5 years. Not only UCLA- and Tegner Score were measured, but also individual information about sports disciplines, frequency and length of activity. This is the first study to give detailed information about sports and activity after cementless medial OUKR. No patient died or was lost to follow-up.

\section{Conclusions}

This study demonstrates that patients treated with cementless OUKR achieve high activity levels after surgery. Furthermore, patients seem to participate in the same sports activities than before onset of OA. Cementless OUKR allows fast recovery and a high return-toactivity rate. Quality of life was excellent compared to the healthy reference group.

\section{Abbreviations}

ACL: Anterior Cruciate Ligament; OA: Osteoarthritis; OUKR: Oxford Unicompartimental Knee Replacement; PFA: Patello Femoral Arthroplasty; PFJ: Patello Femoral Joint; UKR: Unicompartimental Knee Replacement

\section{Acknowledgements}

Not applicable

\section{Funding}

This research did not receive any specific grant from funding agencies in the public, commercial, or not-for-profit sectors.

\section{Availability of data and materials}

All data generated or analysed during this study will be provided by the corresponding author TG on request.

\section{Authors' contributions}

$\mathrm{BP}, \mathrm{IB}, \mathrm{TG}$ and $\mathrm{SH}$ contributed to study conception and design. BP, IB, TR and TW contributed to the acquisition of data. BP, IB, TG, TW and SH contributed to analysis and interpretation of data. BP, IB, TG drafted the manuscript. All authors critically revised the manuscript. All authors read and approved the final manuscript.

\section{Ethics approval and consent to participate}

The institutional review board of the University of Heidelberg approved all procedures of this study (S-546/2013). Written consent was obtained from all patients included in this study.

\section{Consent for publication}

Not applicable

\section{Competing interests}

TG and TW received payment from Zimmer Biomet as a Principal Investigator outside of this submitted work.

\section{Publisher's Note}

Springer Nature remains neutral with regard to jurisdictional claims in published maps and institutional affiliations.

Received: 27 June 2017 Accepted: 29 November 2017

Published online: 17 January 2018

References

1. No authors listed (c) (2013) National Joint Registry for England, Wales and Northern Ireland: 10th Annual Report 2013.. http://www.njrcentre.org.uk/ njrcentre/Portals/0/Documents/England/Reports/10th_annual_report/ NJR\%2010th\%20Annual\%20Report\%202013\%20B.pdf.

2. Naal FD, Fischer M, Preuss $A$, et al. Return to sports and recreational activity after unicompartmental knee arthroplasty. Am J Sports Med. 2007:35(10):1688-95.

3. Pietschmann MF, Wohlleb L, Weber P et al. (2013) Sports activities after medial unicompartmental knee arthroplasty Oxford III-what can we expect? Int Orthop 37 (1):31-37.

4. Amin AK, Patton JT, Cook RE, et al. Unicompartmental or total knee arthroplasty?: results from a matched study. Clin Orthop Relat Res. 2006 451:101-6.

5. Brown NM, Sheth NP, Davis K, et al. Total knee arthroplasty has higher postoperative morbidity than unicompartmental knee arthroplasty: a multicenter analysis. J Arthroplast. 2012;27(8 Suppl):86-90.

6. Laurencin $C T$, Zelicof SB, Scott RD, et al. Unicompartmental versus total knee arthroplasty in the same patient. A comparative study. Clin Orthop Relat Res. 1991;273:151-6.

7. Price AJ, Waite JC, Svard U. Long-term clinical results of the medial Oxford unicompartmental knee arthroplasty. Clin Orthop Relat Res. 2005:435:171-80

8. Pandit H, Jenkins C, Barker K, et al. The Oxford medial unicompartmental knee replacement using a minimally-invasive approach. J Bone Joint Surg Br. 2006;88(1):54-60

9. Lisowski LA, van den Bekerom MP, Pilot $P$, et al. Oxford phase 3 unicompartmental knee arthroplasty: medium-term results of a minimally invasive surgical procedure. Knee Surg Sports Traumatol Arthrosc. 2011; 19(2):277-84.

10. Price AJ, Dodd CA, Svard UG, et al. Oxford medial unicompartmental knee arthroplasty in patients younger and older than 60 years of age. J Bone Joint Surg Br. 2005;87(11):1488-92.

11. Walker T, Streit J, Gotterbarm T, et al. Sports, physical activity and patientreported outcomes after medial Unicompartmental knee Arthroplasty in young patients. J Arthroplasty. 2015;30(11):1911-6. https://doi.org/10.1016/j. arth.2015.05.031

12. Kort NP, van Raay JJ, van Horn JJ. The Oxford phase III unicompartmental knee replacement in patients less than 60 years of age. Knee Surg Sports Traumatol Arthrosc. 2007;15(4):356-60.

13. Price AJ, Svard U. A second decade lifetable survival analysis of the Oxford unicompartmental knee arthroplasty. Clin Orthop Relat Res. 2011:469(1):174-9.

14. Pandit $H$, Jenkins C, Beard DJ, et al. Cementless Oxford unicompartmental knee replacement shows reduced radiolucency at one year. J Bone Joint Surg Br. 2009;91(2):185-9.

15. Seeger JB, Haas $D$, Jager $S$, et al. Extended sagittal saw cut significantly reduces fracture load in cementless unicompartmental knee arthroplasty 
compared to cemented tibia plateaus: an experimental cadaver study. Knee Surg Sports Traumatol Arthrosc. 2012;20(6):1087-91.

16. Liddle $A D$, Pandit $H G$, Jenkins $C$, et al. Valgus subsidence of the tibial component in cementless Oxford unicompartmental knee replacement. Bone Joint J. 2014;96-B(3):345-9.

17. Akan B, Karaguven D, Guclu B, et al. Cemented versus Uncemented Oxford Unicompartmental knee Arthroplasty: is there a difference? Adv Orthop. 2013;2013:245915.

18. Liddle AD, Pandit H, O'Brien S, et al. Cementless fixation in Oxford unicompartmental knee replacement: a multicentre study of 1000 knees. Bone Joint J. 2013;95-B(2):181-7.

19. Pandit $H$, Liddle $A D$, Kendrick BJ, et al. Improved fixation in cementless unicompartmental knee replacement: five-year results of a randomized controlled trial. J Bone Joint Surg Am. 2013;95(15):1365-72.

20. Rothwell A, Hobbs, T., Frampton, C. (2012) The New Zealand joint registry. Thirteen year report: New Zeeland Orthopaedic Association. January 1999 to December $2011 \mathrm{http}: / /$ zzoa.org.nz/system/files/ NJR\%2013\%20Year\%20Report.pdf.

21. Panzram B, Bertlich I, Reiner T, et al. Results after Cementless medial Oxford Unicompartmental knee replacement - incidence of radiolucent lines. PLoS One. 2017;12(1):e0170324.

22. Pandit $\mathrm{H}$, Jenkins $\mathrm{C}$, Gill HS, et al. Unnecessary contraindications for mobilebearing unicompartmental knee replacement. J Bone Joint Surg Br. 2011; 93(5):622-8.

23. Goodfellow JW, Kershaw CJ, Benson MK, et al. The Oxford knee for unicompartmental osteoarthritis. The first 103 cases. J Bone Joint Surg Br. 1988;70(5):692-701.

24. Chang MJ, Kim SH, Kang YG, et al. Activity levels and participation in physical activities by Korean patients following total knee arthroplasty. BMC Musculoskelet Disord. 2014;15:240.

25. Witjes S, Gouttebarge V, Kuijer PP, et al. Return to sports and physical activity after Total and Unicondylar knee Arthroplasty: a systematic review and meta-analysis. Sports Med. 2016;46(2):269-92.

26. Robertsson O, Knutson K, Lewold S, et al. The routine of surgical management reduces failure after unicompartmental knee arthroplasty. J Bone Joint Surg Br. 2001;83(1):45-9.

27. Rees JL, Price AJ, Beard DJ, et al. Minimally invasive Oxford unicompartmental knee arthroplasty: functional results at 1 year and the effect of surgical inexperience. Knee. 2004;11(5):363-7.

28. Fisher N, Agarwal M, Reuben SF, et al. Sporting and physical activity following Oxford medial unicompartmental knee arthroplasty. Knee. 2006;13(4):296-300.

29. Hopper GP, Leach WJ. Participation in sporting activities following knee replacement: total versus unicompartmental. Knee Surg Sports Traumatol Arthrosc. 2008;16(10):973-9.

30. Walker T, Gotterbarm T, Bruckner T, et al. Return to sports, recreational activity and patient-reported outcomes after lateral unicompartmental knee arthroplasty. Knee Surg Sports Traumatol Arthrosc. 2015;23(11):3281-7.

31. Price AJ, Webb J, Topf $\mathrm{H}$, et al. Rapid recovery after oxford unicompartmental arthroplasty through a short incision. J Arthroplast. 2001;16(8):970-6.

32. Jahnke A, Mende JK, Maier GS, et al. Sports activities before and after medial unicompartmental knee arthroplasty using the new Heidelberg sports activity score. Int Orthop. 2015;39(3):449-54.

33. Felts $E$, Parratte $S$, Pauly $V$, et al. Function and quality of life following medial unicompartmental knee arthroplasty in patients 60 years of age or younger. Orthop Traumatol Surg Res. 2010;96(8):861-7.

34. Innmann MM, Weiss S, Andreas F, et al. Sports and physical activity after cementless total hip arthroplasty with a minimum follow-up of 10 years Scand J Med Sci Sports. 2016;26(5):550-6. https://doi.org/10.1111/sms.12482.

35. Wylde V, Blom A, Dieppe P, et al. Return to sport after joint replacement. J Bone Joint Surg Br. 2008;90(7):920-3.

36. Swanson EA, Schmalzried TP, Dorey FJ. Activity recommendations after total hip and knee arthroplasty: a survey of the American Association for hip and Knee Surgeons. J Arthroplast. 2009;24(6 Suppl):120-6.

37. Ollivier M, Frey $S$, Parratte $S$, et al. Does impact sport activity influence total hip arthroplasty durability? Clin Orthop Relat Res. 2012;470(11):3060-6.

38. Price AJ, Short A, Kellett C, et al. Ten-year in vivo wear measurement of a fully congruent mobile bearing unicompartmental knee arthroplasty. J Bone Joint Surg Br. 2005;87(11):1493-7.

39. Kendrick BJ, Longino D, Pandit $\mathrm{H}$, et al. Polyethylene wear in Oxford unicompartmental knee replacement: a retrieval study of 47 bearings. J Bone Joint Surg Br. 2010;92(3):367-73.
40. Bassey EJ, Ramsdale SJ. Increase in femoral bone density in young women following high-impact exercise. Osteoporos Int. 1994;4(2):72-5.

41. Whalen RT, Carter DR, Steele CR. Influence of physical activity on the regulation of bone density. J Biomech. 1988;21(10):825-37.

42. Scerpella TA, Davenport M, Morganti CM, et al. Dose related association of impact activity and bone mineral density in pre-pubertal girls. Calcif Tissue Int. 2003;72(1):24-31.

\section{Submit your next manuscript to BioMed Central and we will help you at every step:}

- We accept pre-submission inquiries

- Our selector tool helps you to find the most relevant journal

- We provide round the clock customer support

- Convenient online submission

- Thorough peer review

- Inclusion in PubMed and all major indexing services

- Maximum visibility for your research

Submit your manuscript at www.biomedcentral.com/submit
Biomed Central 